\title{
Translation and modulation invariant Hilbert spaces
}

\author{
Joachim Toft ${ }^{1}\left[\right.$ ) Anupam Gumber ${ }^{2} \cdot$ Ramesh Manna $^{3} \cdot$ P. K. Ratnakumar ${ }^{4}$
}

Received: 9 April 2020 / Accepted: 22 June 2021 / Published online: 19 July 2021

(c) The Author(s) 2021

\begin{abstract}
Let $\mathcal{H}$ be a Hilbert space of distributions on $\mathbf{R}^{d}$ which contains at least one non-zero element of the Feichtinger algebra $S_{0}$ and is continuously embedded in $\mathscr{D}^{\prime}$. If $\mathcal{H}$ is translation and modulation invariant, also in the sense of its norm, then we prove that $\mathcal{H}=L^{2}$, with the same norm apart from a multiplicative constant.
\end{abstract}

Keywords Modulation spaces · Feichtinger's minimization principle

Mathematics Subject Classification 46C15 · 46C05 - 42B35

\section{Introduction}

In the paper we show that any Hilbert space of distributions on $\mathbf{R}^{d}$ which is translation and modulation invariant with respect to the norms, agrees with $L^{2}\left(\mathbf{R}^{d}\right)$. These considerations are strongly linked with Feichtinger's minimization property, which

Communicated by Adrian Constantin.

$凶$ Joachim Toft

joachim.toft@lnu.se

Anupam Gumber

anupamgumber@iisc.ac.in

Ramesh Manna

rameshmanna@niser.ac.in

P. K. Ratnakumar

ratnapk@hri.res.in

1 Department of Mathematics, Linnæus University, Växjö, Sweden

2 Department of Mathematics, Indian Institute of Science, Bangalore 560012, India

3 School of Mathematical Sciences, National Institute of Science Education and Research Bhubaneswar, HBNI, Jatni 752050, India

4 Harish-Chandra Research Institute (HBNI), Chhatnag Road, Jhunsi, Allahabad, Uttar Pradesh 211019, India 
shows that the Feichtinger algebra $S_{0}\left(\mathbf{R}^{d}\right)$, which is the same as the modulation space $M^{1,1}\left(\mathbf{R}^{d}\right)$, is the smallest non-trivial Banach space of tempered distributions which is norm invariant under translations and modulations. Our investigations may therefore be considered as a Hilbert space analogue of those investigations which lead to Feichtinger's minimization property.

We remark that the search for the smallest Banach space possessing such norm invariance properties, seems to be the main reason that Feichtinger led to introduce and investigate $S_{0}\left(\mathbf{R}^{d}\right)$ and in its prolongation the foundation of classical modulation spaces (see [2]). The space $S_{0}\left(\mathbf{R}^{d}\right)=M^{1,1}\left(\mathbf{R}^{d}\right)$ is small in the sense that it is contained in every Lebesgue space $L^{p}\left(\mathbf{R}^{d}\right)$ and its Fourier image, $p \in[1, \infty]$ (cf. [2,5,9] and the references therein).

On the contrary, the modulation space $M^{\infty, \infty}\left(\mathbf{R}^{d}\right)$, which is the dual of $M^{1,1}\left(\mathbf{R}^{d}\right)$, contains all these Lebesgue and Fourier Lebesgue spaces. By straight-forward arguments (see Proposition A.1) it follows that for a translation and modulation invariant Banach space $\mathcal{B}$ of tempered distributions which contains at least one non-zero element in $S_{0}\left(\mathbf{R}^{d}\right)$, we have

$$
S_{0}\left(\mathbf{R}^{d}\right)=M^{1,1}\left(\mathbf{R}^{d}\right) \subseteq \mathcal{B} \subseteq M^{\infty, \infty}\left(\mathbf{R}^{d}\right)
$$

Here the first inclusion is a reformulation of the Feichtinger's minimization property (in the unweighted case). We refer to Sect. 1 and $[5,6]$ for notations and some facts on distributions.

Feichtinger's minimization property has been extended in different ways, e.g. to weighted spaces (see e.g. [5, Chapter 12]), and to the quasi-Banach situation (see e.g. [9]). At the same time minimization property has been applied e.g. in non-uniform samplings, and for deducing sharp Schatten-von Neumann and nuclear results for operators with kernels in modulation spaces (see e.g. [9]). Note also that translation and modulation invariant Banach spaces are important in Gabor analysis, e.g. when searching for suitable windows for Gabor frames. It also seems necessary for a Banach space to be translation and modulation invariant, if it should be conveniently discretizable by Gabor expansions (see e.g. [5] and the references therein).

In our investigations we do not present any such weighted analogies in the Hilbert space case. On the other hand, we consider translation and modulation invariant Banach spaces, $\mathcal{B}$, which are continuously embedded in $\mathscr{D}^{\prime}\left(\mathbf{R}^{d}\right)$, the set of all distributions on $\mathbf{R}^{d}$. As a first step we prove in Proposition 1.5 that any such $\mathcal{B}$ is continuously embedded in $\mathscr{S}^{\prime}\left(\mathbf{R}^{d}\right)$, the set of all tempered distributions on $\mathbf{R}^{d}$. Note that the original approaches in $[2,5]$ have the more restrictive assumption that these $\mathcal{B}$ are continuously embedded in the subspace $\mathscr{S}^{\prime}\left(\mathbf{R}^{d}\right)$ of $\mathscr{D}^{\prime}\left(\mathbf{R}^{d}\right)$.

\section{Translation and modulation invariant Hilbert spaces}

In this section we first recall the definition of translation and modulation invariant spaces. Thereafter we consider such spaces which at the same time are Hilbert spaces of distributions on $\mathbf{R}^{d}$. We show some features on how differentiations and multipli- 
cations by polynomials of such spaces behave in the inner product of such Hilbert spaces. In the end we show that such Hilbert spaces agree with $L^{2}\left(\mathbf{R}^{d}\right)$.

The definition of translation and modulation invariant Banach spaces is given in the following (cf. [2,5]).

Definition 1.1 Let $\mathcal{B}$ be a Banach space which is continuously embedded in $\mathscr{D}^{\prime}\left(\mathbf{R}^{d}\right)$. Then $\mathcal{B}$ is called translation and modulation invariant, if $y \mapsto f(y-x) e^{i\langle y, \xi\rangle}$ belongs to $\mathcal{B}$ and

$$
\left\|f(\cdot-x) e^{i\langle\cdot, \xi\rangle}\right\|_{\mathcal{B}}=\|f\|_{\mathcal{B}}
$$

for every $f \in \mathcal{B}$ and $x, \xi \in \mathbf{R}^{d}$.

Remark 1.2 We identify $\mathscr{S}^{\prime}\left(\mathbf{R}^{d}\right)$ as a subspace of $\mathscr{D}^{\prime}\left(\mathbf{R}^{d}\right)$ in the same way as in [6]. In particular it follows that $f \in \mathscr{D}^{\prime}\left(\mathbf{R}^{d}\right)$ belongs to $\mathscr{S}^{\prime}\left(\mathbf{R}^{d}\right)$, if and only if there is a constant $C>0$ and semi-norm $\|\cdot\|$ on $\mathscr{S}\left(\mathbf{R}^{d}\right)$ such that

$$
|\langle f, \psi\rangle| \leq C\|\psi\|
$$

for every $\psi \in C_{0}^{\infty}\left(\mathbf{R}^{d}\right)$. For such $f$, there is a unique $f_{0} \in \mathscr{S}^{\prime}\left(\mathbf{R}^{d}\right)$ such that $\langle f, \psi\rangle=\left\langle f_{0}, \psi\right\rangle$ for every $\psi \in C_{0}^{\infty}\left(\mathbf{R}^{d}\right)$. (See [6][Chapter VII].) Then we identify $f$ with $f_{0}$, and thereby consider $f$ as an element in $\mathscr{S}^{\prime}\left(\mathbf{R}^{d}\right)$.

Our main result is the following.

Theorem 1.3 Let $\mathcal{H}$ be a translation and modulation invariant Hilbert space, continuously embedded in $\mathscr{D}^{\prime}\left(\mathbf{R}^{d}\right)$ and contains at least one element of $M^{1,1}\left(\mathbf{R}^{d}\right) \backslash\{0\}$. Then $\mathcal{H}=L^{2}\left(\mathbf{R}^{d}\right)$ with

$$
\|f\|_{\mathcal{H}}=c \cdot\|f\|_{L^{2}\left(\mathbf{R}^{d}\right)},
$$

for some constant $c>0$ which is independent of $f \in \mathcal{H}=L^{2}\left(\mathbf{R}^{d}\right)$.

We observe that $L^{2}\left(\mathbf{R}^{d}\right)$ is the same as the modulation space $M^{2,2}\left(\mathbf{R}^{d}\right)$ (see [2]). Hence the analogy of $(0.1)$ in the Hilbert space case is that for translation and modulation invariant Hilbert spaces $\mathcal{H}$ one has

$$
M^{2,2}\left(\mathbf{R}^{d}\right)=\mathcal{H}
$$

Remark 1.4 It is obvious that the constant $c$ in (1.1) can be evaluated by

$$
c=\left(\|f\|_{L^{2}\left(\mathbf{R}^{d}\right)}\right)^{-1}\|f\|_{\mathcal{H}}
$$

for any fixed $f \in \mathcal{H} \backslash\{0\}$.

We need some preparations for the proof of Theorem 1.3. First we note that the restriction of the $L^{2}$ scalar product $(\cdot, \cdot)_{L^{2}}$ on $\mathscr{S}\left(\mathbf{R}^{d}\right) \times \mathscr{S}\left(\mathbf{R}^{d}\right)$ is uniquely extendable to a continuous sesqui-linear form on $\mathscr{S}\left(\mathbf{R}^{d}\right) \times \mathscr{S}^{\prime}\left(\mathbf{R}^{d}\right)$ and that the dual of $\mathscr{S}\left(\mathbf{R}^{d}\right)$ can be identified by $\mathscr{S}^{\prime}\left(\mathbf{R}^{d}\right)$ through this extension.

As a consequence of the following proposition we have that any translation and modulation invariant Banach space on $\mathbf{R}^{d}$ is continuously embedded in $\mathscr{S}^{\prime}\left(\mathbf{R}^{d}\right)$. 
Proposition 1.5 Let $\mathcal{B}$ be a Banach space, continuously embedded in $\mathscr{D}^{\prime}\left(\mathbf{R}^{d}\right)$ such that $y \mapsto f(y-x)$ belongs to $\mathcal{B}$ for every $x \in \mathbf{R}^{d}$ and

$$
\|f(\cdot-x)\|_{\mathcal{B}} \leq C(1+|x|)^{r}\|f\|_{\mathcal{B}},
$$

for some constants $C, r>0$ which are independent of $f \in \mathcal{B}$ and $x \in \mathbf{R}^{d}$. Then $\mathcal{B}$ is continuously embedded in $\mathscr{S}^{\prime}\left(\mathbf{R}^{d}\right)$.

Proposition 1.5 follows by similar arguments as in the proof of Bochner-Schwartz theorem for positive definite distributions (see e.g. [7] and the references therein). In order to be self contained we give a proof in Appendix A.

Remark 1.6 By Proposition 1.5 it follows that results on Feichtinger's minimization property in e.g. [2,5,9], still hold true with relaxed assumptions that the involved Banach spaces are embedded in $\mathscr{D}^{\prime}\left(\mathbf{R}^{d}\right)$ instead of its subspace $\mathscr{S}^{\prime}\left(\mathbf{R}^{d}\right)$.

For a translation and modulation invariant Banach space $\mathcal{B}$ in Definition 1.1, there is also a maximization property, analogous to Feichtinger's minimization property (see Proposition A.1 in Appendix A). In the unweighted case, the largest possible such $\mathcal{B}$ is given by $S_{0}^{\prime}\left(\mathbf{R}^{d}\right)=M^{\infty, \infty}\left(\mathbf{R}^{d}\right)$ in the Banach-Gelfand triple $\left(S_{0}\left(\mathbf{R}^{d}\right), L^{2}\left(\mathbf{R}^{d}\right), S_{0}^{\prime}\left(\mathbf{R}^{d}\right)\right.$ ) studied in [4] (see Appendix A for the definition of $\left.M^{\infty, \infty}\left(\mathbf{R}^{d}\right)\right)$.

As a consequence of Proposition 1.5 we have that $\mathcal{H}$ in Theorem 1.3 is contained in $\mathscr{S}^{\prime}\left(\mathbf{R}^{d}\right)$. Since $\mathcal{H}$ contains at least one element in $M^{1,1}\left(\mathbf{R}^{d}\right)$ we get the more refined

$$
\mathscr{S}\left(\mathbf{R}^{d}\right) \subseteq M^{1,1}\left(\mathbf{R}^{d}\right) \subseteq \mathcal{H} \subseteq M^{\infty, \infty}\left(\mathbf{R}^{d}\right)
$$

with continuous inclusions, by Proposition A.1 and Feichtinger's minimization property. In particular, the standard Gaussian $h_{0}(x)=\pi^{-\frac{d}{4}} e^{-\frac{1}{2}|x|^{2}}$ belongs to $\mathcal{H}$.

For the proof of Theorem 1.3, we also need some properties on Hermite functions. Recall that the Hermite function $h_{\alpha}$ of order $\alpha \in \mathbf{N}^{d}$ on $\mathbf{R}^{d}$ is defined by

$$
h_{\alpha}(x)=\pi^{-\frac{d}{4}}(-1)^{|\alpha|}\left(2^{|\alpha|} \alpha !\right)^{-\frac{1}{2}} e^{\frac{1}{2}|x|^{2}}\left(\partial^{\alpha} e^{-|x|^{2}}\right), \quad x \in \mathbf{R}^{d}, \alpha \in \mathbf{N}^{d} .
$$

It is well-known that $\left\{h_{\alpha}\right\}_{\alpha \in \mathbf{N}^{d}}$ is an orthonormal basis for $L^{2}\left(\mathbf{R}^{d}\right)$, and a basis for $\mathscr{S}\left(\mathbf{R}^{d}\right)$ (see e.g. [7,8]).

We may pass from one Hermite function to another by successively applying the annihilation and creation operators, which are given by

$$
\mathrm{A}_{j}=\frac{1}{\sqrt{2}}\left(x_{j}+\frac{\partial}{\partial x_{j}}\right) \quad \text { and } \quad \mathrm{C}_{j}=\frac{1}{\sqrt{2}}\left(x_{j}-\frac{\partial}{\partial x_{j}}\right),
$$

respectively, $j=1, \ldots, d$. It is then well-known that if $e_{j}$ is the $j$ th vector in the standard basis in $\mathbf{R}^{d}$, then

$$
\mathrm{A}_{j} h_{\alpha}= \begin{cases}\sqrt{\alpha_{j}} h_{\alpha-e_{j}}, & \alpha_{j} \geq 1 \\ 0, & \alpha_{j}=0\end{cases}
$$


and

$$
\mathrm{C}_{j} h_{\alpha}=\sqrt{\alpha_{j}+1} h_{\alpha+e_{j}}, \quad \alpha \in \mathbf{N}^{d} .
$$

(Cf. e.g. [1].) This implies

$$
\mathrm{A}^{\alpha} h_{\alpha}=\alpha !^{\frac{1}{2}} h_{0}, \quad \text { and } \quad \mathrm{C}^{\alpha} h_{0}=\alpha !^{\frac{1}{2}} h_{\alpha} \text {, }
$$

where

$$
\mathrm{A}^{\alpha}=\prod_{j=1}^{d} \mathrm{~A}_{j}^{\alpha_{j}}, \quad \text { and } \quad \mathrm{C}^{\alpha}=\prod_{j=1}^{d} \mathrm{C}_{j}^{\alpha_{j}}, \quad \alpha=\left(\alpha_{1}, \ldots, \alpha_{d}\right) .
$$

Furthermore,

$$
\mathrm{A}^{\beta} h_{\alpha}=0 \text { when } \alpha_{j}<\beta_{j} \text { for some } j \in\{1, \ldots, d\} .
$$

We have now the following lemma.

Lemma 1.7 Let $\mathcal{H}$ be a translation and modulation invariant Hilbert space on $\mathbf{R}^{d}$. Then the following is true:

(1) for every $f, g \in \mathcal{H}$ and $x, \xi \in \mathbf{R}^{d}$ it holds

$$
(f(\cdot-x), g)_{\mathcal{H}}=(f, g(\cdot+x))_{\mathcal{H}}
$$

and

$$
\left(f \cdot e^{-i\langle\cdot \xi\rangle}, g\right)_{\mathcal{H}}=\left(f, g \cdot e^{i\langle\cdot, \xi\rangle}\right)_{\mathcal{H}}
$$

(2) $\left(\partial^{\alpha} f, g\right)_{\mathcal{H}}=\left(f,(-\partial)^{\alpha} g\right)_{\mathcal{H}}$ and $\left(x^{\alpha} f, g\right)_{\mathcal{H}}=\left(f, x^{\alpha} g\right)_{\mathcal{H}}$ for every $f, g \in$ $\mathscr{S}\left(\mathbf{R}^{d}\right)$ and $\alpha \in \mathbf{N}^{d}$.

We observe that (2) in the previous lemma gives

$$
\left(f, \mathrm{C}_{j} g\right)_{\mathcal{H}}=\left(\mathrm{A}_{j} f, g\right)_{\mathcal{H}}, \quad f, g \in \mathscr{S}\left(\mathbf{R}^{d}\right), j=1, \ldots, d .
$$

Proof We have by Definition 1.1

$$
\left\|f(\cdot-x) e^{-i\langle\cdot, \xi\rangle}\right\|_{\mathcal{H}}^{2}=\|f\|_{\mathcal{H}}^{2}
$$

which by polarization gives

$$
\left(f(\cdot-x) e^{-i\langle\cdot, \xi\rangle}, g(\cdot-x) e^{-i\langle\cdot, \xi\rangle}\right)_{\mathcal{H}}=(f, g)_{\mathcal{H}},
$$

when $f, g \in \mathcal{H}$ and $x, \xi \in \mathbf{R}^{d}$. This gives (1).

The assertion (2) follows by applying $\partial_{x}^{\alpha}$ and $\partial_{\xi}^{\alpha}$ on (1.9) and (1.10) with $f, g \in$ $\mathscr{S}\left(\mathbf{R}^{d}\right)$, and then putting $x=\xi=0$. 
Proof of Theorem 1.3 Suppose that $\alpha, \beta \in \mathbf{N}^{d}$ are such that $\beta_{j}>\alpha_{j}$ for some $j \in$ $\{1, \ldots, d\}$. By (1.11), (1.6) and (1.8) we get

$$
\left(h_{\alpha}, h_{\beta}\right)_{\mathcal{H}}=\beta !^{-\frac{1}{2}}\left(h_{\alpha}, \mathrm{C}^{\beta} h_{0}\right)_{\mathcal{H}}=\beta !^{-\frac{1}{2}}\left(\mathrm{~A}^{\beta} h_{\alpha}, h_{0}\right)_{\mathcal{H}}=0
$$

and

$$
\begin{aligned}
\left\|h_{\alpha}\right\|_{\mathcal{H}}^{2} & =\left(h_{\alpha}, h_{\alpha}\right)_{\mathcal{H}}=\alpha !^{-\frac{1}{2}}\left(h_{\alpha}, \mathrm{C}^{\alpha} h_{0}\right)_{\mathcal{H}} \\
& =\alpha !^{-\frac{1}{2}}\left(\mathrm{~A}^{\alpha} h_{\alpha}, h_{0}\right)_{\mathcal{H}}=\left(h_{0}, h_{0}\right)_{\mathcal{H}}=\left\|h_{0}\right\|_{\mathcal{H}}^{2} .
\end{aligned}
$$

This implies that $\left\{\left\|h_{0}\right\|_{\mathcal{H}}^{-1} h_{\alpha}\right\}_{\alpha \in \mathbf{N}^{d}}$ is an orthonormal system for $\mathcal{H}$.

Hence, if $f \in \mathscr{S}\left(\mathbf{R}^{d}\right)$, then

$$
\|f\|_{\mathcal{H}}^{2}=\left\|\sum_{\alpha \in \mathbf{N}^{d}}\left(f, h_{\alpha}\right)_{L^{2}} h_{\alpha}\right\|_{\mathcal{H}}^{2}=\left\|h_{0}\right\|_{\mathcal{H}}^{2} \sum_{\alpha \in \mathbf{N}^{d}}\left|\left(f, h_{\alpha}\right)_{L^{2}}\right|^{2}=\left\|h_{0}\right\|_{\mathcal{H}}^{2}\|f\|_{L^{2}}^{2}
$$

Since the inclusions

$$
\mathscr{S}\left(\mathbf{R}^{d}\right) \subseteq M^{1,1}\left(\mathbf{R}^{d}\right) \subseteq L^{2}\left(\mathbf{R}^{d}\right)
$$

are continuous and dense (see e.g. [2,5]), it follows that $L^{2}\left(\mathbf{R}^{d}\right)$ is continuously embedded in $\mathcal{H}$, and that (1.1) holds. Furthermore, let from now on the original $\mathcal{H}$ norm be replaced by the equivalent Hilbert norm $f \mapsto\left\|h_{0}\right\|_{\mathcal{H}}^{-1}\|f\|_{\mathcal{H}}$. Then it follows that the inclusion $i: L^{2}\left(\mathbf{R}^{d}\right) \rightarrow \mathcal{H}$ is an isometric injection.

We shall use Hahn-Banach's theorem to prove that the latter map is in fact bijective. Suppose that $\ell$ is a linear continuous form on $\mathcal{H}$ which is zero on $L^{2}\left(\mathbf{R}^{d}\right)$. Then $\ell(f)=\left(f, g_{0}\right)_{\mathcal{H}}$ for some unique $g_{0} \in \mathcal{H}$. We need to prove that $g_{0}=0$.

Since the forms $(\cdot, \cdot)_{L^{2}}$ and $(\cdot, \cdot)_{\mathcal{H}}$ agree on $L^{2}\left(\mathbf{R}^{d}\right)$, which contains $\mathscr{S}\left(\mathbf{R}^{d}\right)$, and since $\mathcal{H} \subseteq \mathscr{S}^{\prime}\left(\mathbf{R}^{d}\right)$, the same extension and duality properties hold true with $(\cdot, \cdot)_{\mathcal{H}}$ in place of $(\cdot, \cdot)_{L^{2}}$ (cf. the paragraph after Remark 1.4). In particular,

$$
(f, g)_{L^{2}}=(f, g)_{\mathcal{H}}, \quad f \in \mathscr{S}\left(\mathbf{R}^{d}\right), g \in \mathscr{S}^{\prime}\left(\mathbf{R}^{d}\right)
$$

We have $g_{0} \in \mathcal{H} \subseteq \mathscr{S}^{\prime}\left(\mathbf{R}^{d}\right)$. Recall that any element in $\mathscr{S}^{\prime}\left(\mathbf{R}^{d}\right)$ has a Hermite series expansion converging in $\mathscr{S}^{\prime}\left(\mathbf{R}^{d}\right)$, whose coefficients are polynomially bounded with respect to their orders (see [7]). Consequently,

$$
g_{0}=\sum_{\alpha \in \mathbf{N}^{d}} c(\alpha) h_{\alpha}
$$


for some $\{c(\alpha)\}_{\alpha \in \mathbf{N}^{d}}$ such that $|c(\alpha)| \leq C(1+|\alpha|)^{N}$ for some constants $C, N>0$. Since $\left(f, g_{0}\right)_{\mathcal{H}}=0$ when $f \in L^{2}\left(\mathbf{R}^{d}\right)$, and that $h_{\alpha} \in \mathscr{S}\left(\mathbf{R}^{d}\right)$ we get

$$
c(\alpha)=\overline{\left(h_{\alpha}, g_{0}\right)_{L^{2}}}=\overline{\left(h_{\alpha}, g_{0}\right)_{\mathcal{H}}}=0,
$$

giving that $g_{0}=0$.

By Hahn-Banach's theorem it follows that $L^{2}\left(\mathbf{R}^{d}\right)$ is dense in $\mathcal{H}$. Since $L^{2}\left(\mathbf{R}^{d}\right)$ is also a closed subset of $\mathcal{H}$ in view of (1.13), it follows that $\mathcal{H}=L^{2}\left(\mathbf{R}^{d}\right)$, and the result follows.

Acknowledgements The first author was supported by Vetenskapsrådet (Swedish Science Council) within the project 2019-04890. The second author is grateful for the support received from NBHM grant (0204/19/2019R\&D-II/10472). The third author is thankful to Indian Institute of Science (C.V. Raman PDF, file no: R(IA)CVR-PDF/2020/224) for the financial support. The fourth author wishes to thank the infosys foundation for their generous financial support to Harish-Chandra Research Institute (HRI), Prayagraj (Allahabad), for inviting foreign experts and facilitating research collaboration.

The authors are also grateful to HRI for the excellent hospitality and research facilities.

Funding Open access funding provided by Linnaeus University.

Open Access This article is licensed under a Creative Commons Attribution 4.0 International License, which permits use, sharing, adaptation, distribution and reproduction in any medium or format, as long as you give appropriate credit to the original author(s) and the source, provide a link to the Creative Commons licence, and indicate if changes were made. The images or other third party material in this article are included in the article's Creative Commons licence, unless indicated otherwise in a credit line to the material. If material is not included in the article's Creative Commons licence and your intended use is not permitted by statutory regulation or exceeds the permitted use, you will need to obtain permission directly from the copyright holder. To view a copy of this licence, visit http://creativecommons.org/licenses/by/4.0/.

\section{Appendix A. Some properties of translation invariant Banach spaces}

In this appendix we give a proof of Proposition 1.5 and discuss maximality of translation and modulation invariant Banach spaces of distributions on $\mathbf{R}^{d}$.

Proof of Proposition 1.5 Let $\langle x\rangle=1+|x|, \varepsilon>0, Q$ be the cube $[0,1+\varepsilon]^{d} \subseteq \mathbf{R}^{d}$ and $0 \leq \varphi \in C_{0}^{\infty}\left(\mathbf{R}^{d}\right)$ be such that

$$
\operatorname{supp} \varphi \subseteq Q \text { and } \sum_{j \in \mathbf{Z}^{d}} \varphi(\cdot-j)=1 .
$$

Since $\mathcal{B}$ is continuously embedded in $\mathscr{D}^{\prime}\left(\mathbf{R}^{d}\right)$, it follows from Chapter II in [6] that there exists a constant $C>0$ and integer $N \geq 0$ such that

$$
\left|\left\langle f, \psi_{0}\right\rangle\right| \leq C\|f\|_{\mathcal{B}} \sum_{|\alpha| \leq N}\left\|\partial^{\alpha} \psi_{0}\right\|_{L^{\infty}(Q)},
$$

for every $\psi_{0} \in C_{0}^{\infty}(Q)$ and $f \in \mathcal{B}$. Here we have also used the fact that a distribution restricted to a compact set has finite order. 
Hence, if $\psi \in C_{0}^{\infty}\left(\mathbf{R}^{d}\right)$, Leibniz' rule gives

$$
\begin{aligned}
\mid\langle f, \psi\rangle) \mid & =\left|\left\langle f, \sum_{j \in \mathbf{Z}^{d}} \psi \cdot \varphi(\cdot-j)\right\rangle\right| \\
& \leq \sum_{j \in \mathbf{Z}^{d}}|\langle f(\cdot+j), \psi(\cdot+j) \varphi\rangle| \\
& \leq C_{1} \sum_{j \in \mathbf{Z}^{d}}\|f(\cdot+j)\|_{\mathcal{B}} \sum_{|\alpha| \leq N}\left\|\partial^{\alpha}(\psi(\cdot+j) \varphi)\right\|_{L^{\infty}(Q)} \\
& \leq C_{2}\|f\|_{\mathcal{B}} \sum_{j \in \mathbf{Z}^{d}} \sum_{|\alpha| \leq N}\langle j\rangle^{r}\left\|\partial^{\alpha}(\psi(\cdot+j) \varphi)\right\|_{L^{\infty}(Q)} \\
& \leq C_{3}\|f\|_{\mathcal{B}} \sum_{j \in \mathbf{Z}^{d}} \sum_{|\alpha|,|\beta| \leq N}\langle j\rangle^{r}\left\|\left(\partial^{\alpha} \psi\right)(\cdot+j)\left(\partial^{\beta} \varphi\right)\right\|_{L^{\infty}(Q)} \\
& \leq C_{3}\|f\|_{\mathcal{B}} \sum_{j \in \mathbf{Z}^{d}|\alpha|,|\beta| \leq N}\left\|\left(\langle\cdot+j\rangle^{r}\left(\partial^{\alpha} \psi\right)(\cdot+j)\right)\left(\langle\cdot\rangle^{r} \partial^{\beta} \varphi\right)\right\|_{L^{\infty}(Q)}\left(\sum(\cdot)\right. \\
& \leq C_{3} \cdot C_{\varphi}\|f\|_{\mathcal{B}}\|\psi\|,
\end{aligned}
$$

for some semi-norm $\|\cdot\|$ in $\mathscr{S}\left(\mathbf{R}^{d}\right)$, and some constants $C_{1}, C_{2}$ and $C_{3}$ which are independent of $f \in \mathcal{B}$ and $\psi$. Here $C_{\varphi}$ depends only on $\varphi$, and we have used

$$
\langle x+y\rangle \leq\langle x\rangle \cdot\langle y\rangle .
$$

Hence,

$$
|\langle f, \psi\rangle| \leq C\|f\|_{\mathcal{B}}\|\psi\|
$$

for some constant $C$ which is independent of $f \in \mathcal{B}$ and $\psi \in C_{0}^{\infty}\left(\mathbf{R}^{d}\right)$. By Remark 1.2 and (A.1) it follows that $\mathcal{B}$ is continuously embedded in $\mathscr{S}^{\prime}\left(\mathbf{R}^{d}\right)$.

Next we prove the maximization property for translation and modulation invariant Banach spaces. Let $\omega \in L_{l o c}^{\infty}\left(\mathbf{R}^{2 d} ; \mathbf{R}_{+}\right)$be such that

$$
\omega(x+y, \xi+\eta) \leq C \omega(x, \xi)(1+|y|+|\eta|)^{r}
$$

for some constants $C, r>0$ which are independent of $x, y, \xi, \eta \in \mathbf{R}^{d}$. Also let $\phi \in \mathscr{S}\left(\mathbf{R}^{d}\right) \backslash\{0\}$. Then the modulation space $M_{(1 / \omega)}^{\infty, \infty}\left(\mathbf{R}^{d}\right)$ is given by

$$
M_{(1 / \omega)}^{\infty, \infty}\left(\mathbf{R}^{d}\right)=\left\{f \in \mathscr{S}^{\prime}\left(\mathbf{R}^{d}\right) ;\left\|\left(V_{\phi} f\right) / \omega\right\|_{L^{\infty}}<\infty\right\} .
$$


Here $V_{\phi} f$ is the short-time Fourier transform of $f \in \mathscr{S}^{\prime}\left(\mathbf{R}^{d}\right)$, given by

$$
V_{\phi} f(x, \xi) \equiv(2 \pi)^{-\frac{d}{2}}\left(f, \phi(\cdot-x) e^{i\langle\cdot, \xi\rangle}\right)_{L^{2}}
$$

The Banach space topology in $M_{(1 / \omega)}^{\infty, \infty}\left(\mathbf{R}^{d}\right)$ is then given by the norm

$$
\|f\|_{M_{(1 / \omega)}^{\infty, \infty}}=\left\|\left(V_{\phi} f\right) / \omega\right\|_{L^{\infty}}
$$

(Cf. [2,3,5].) We set $M^{\infty, \infty}=M_{(\omega)}^{\infty, \infty}$ when $\omega=1$.

For other choices of $\omega$ satisfying (A.2), the $L^{2}$ scalar product $(\cdot, \cdot)_{L^{2}}$ on $\mathscr{S}\left(\mathbf{R}^{d}\right) \times$ $\mathscr{S}\left(\mathbf{R}^{d}\right)$ is uniquely extendable to a continuous sesqui-linear form on $M_{(\omega)}^{1,1}\left(\mathbf{R}^{d}\right) \times$ $M_{(1 / \omega)}^{\infty, \infty}\left(\mathbf{R}^{d}\right)$, where $M_{(\omega)}^{1,1}\left(\mathbf{R}^{d}\right)$ is the weighted version of $M^{1,1}\left(\mathbf{R}^{d}\right)$ with respect to $\omega$. Furthermore, $M_{(1 / \omega)}^{\infty, \infty}\left(\mathbf{R}^{d}\right)$ is the dual of $M_{(\omega)}^{1,1}\left(\mathbf{R}^{d}\right)$ with respect to this product (cf. $[2,5])$.

Proposition A.1 Let $\omega \in L_{\text {loc }}^{\infty}\left(\mathbf{R}^{2 d} ; \mathbf{R}_{+}\right)$satisfies (A.2). Let $\mathcal{B}$ be a translation and modulation invariant Banach space which is continuously embedded in $\mathscr{D}^{\prime}\left(\mathbf{R}^{d}\right)$ and contains at least one element of $M_{(\omega)}^{1,1}\left(\mathbf{R}^{d}\right) \backslash\{0\}$. Suppose that for some constant $C>0$ it holds

$$
\left\|f(\cdot+x) e^{-i\langle\cdot, \xi\rangle}\right\|_{\mathcal{B}} \leq C \omega(x, \xi)\|f\|_{\mathcal{B}}, \quad f \in \mathcal{B}, x, \xi \in \mathbf{R}^{d}
$$

Then $\mathcal{B}$ is continuously embedded in $M_{(1 / \omega)}^{\infty, \infty}\left(\mathbf{R}^{d}\right)$.

Proof By Proposition 1.5 it follows that $\mathcal{B}$ is continuously embedded in $\mathscr{S}^{\prime}\left(\mathbf{R}^{d}\right)$. Hence, for any fixed $\phi \in \mathscr{S}\left(\mathbf{R}^{d}\right) \backslash 0$, the map

$$
f \mapsto(f, \phi)_{L^{2}}
$$

is linear and continuous from $\mathcal{B}$ to $\mathbf{C}$, because $\mathcal{B}$ is continuously embedded in $\mathscr{S}^{\prime}\left(\mathbf{R}^{d}\right)$, giving that

$$
\left|(f, \phi)_{L^{2}}\right| \leq C_{\phi}\|f\|_{\mathcal{B}}, \quad f \in \mathcal{B} .
$$

This gives

$$
\begin{aligned}
\left|V_{\phi} f(x, \xi)\right|=\left|\left(f(\cdot+x) e^{-i\langle\cdot, \xi\rangle}, \phi\right)\right| & \leq C_{\phi}\left\|f(\cdot+x) e^{-i\langle\cdot, \xi\rangle}\right\|_{\mathcal{B}} \\
& \leq C_{\phi} C \omega(x, \xi)\|f\|_{\mathcal{B}},
\end{aligned}
$$

which shows that

$$
\left|V_{\phi} f(x, \xi) / \omega(x, \xi)\right| \leq C\|f\|_{\mathcal{B}}
$$


for some constant $C>0$. Hence

$$
\left|\left(V_{\phi} f\right) / \omega\right| \in L^{\infty}\left(\mathbf{R}^{2 d}\right),
$$

which implies $f \in M_{(1 / \omega)}^{\infty, \infty}\left(\mathbf{R}^{d}\right)$. Furthermore, (A.3) shows that

$$
\|f\|_{M_{(1 / \omega)}^{\infty, \infty}} \leq C\|f\|_{\mathcal{B}}, \quad f \in \mathcal{B} .
$$

Consequently, $\mathcal{B}$ is continuously embedded in $M_{(1 / \omega)}^{\infty, \infty}\left(\mathbf{R}^{d}\right)$, and the assertion follows.

\section{References}

1. Bargmann, V.: On a Hilbert space of analytic functions and an associated integral transform. Pure Appl. Math 14, 187-214 (1961)

2. Feichtinger, H.G.: Modulation spaces on locally compact abelian groups. Technical report, University of Vienna, Vienna, 1983; also in: M. Krishna, R. Radha, S. Thangavelu (Eds) Wavelets and their applications, Allied Publishers Private Limited, NewDelhi Mumbai Kolkata Chennai Hagpur Ahmedabad Bangalore Hyderbad Lucknow, 2003, pp. 99-140

3. Feichtinger, H.G.: Modulation spaces: Looking back and ahead. Theory Signal Image Process. 5, 109_ 140 (2006)

4. Feichtinger, H., Luef, F., Cordero, E.: Banach Gelfand triples for Gabor analysis, in: L. Rodino, M. W. Wong (Eds), Pseudo-Differential Operators, Quantization and Signals, Lecture notes in math. 1949, Springer, Berlin Heidelberg, 2008, pp. 1-33

5. Gröchenig, K.: Foundations of Time-Frequency Analysis, Birkhäuser, Boston, 2001

6. Hörmander,L.: The Analysis of Linear Partial Differential Operators, vol I, Springer-Verlag, Berlin Heidelberg NewYork Tokyo, 1983

7. Reed, M., Simon, B.: Methods of Modern Mathematical Physics. Academic Press, London New York (1979)

8. Thangavelu, S.: Lectures on Hermite and Laguerre expansions, Mathematical Notes 42, Princeton University Press, Princeton, N.J., 1993

9. Toft, J.: Schatten properties, nuclearity and minimality of phase shift invariant spaces. Comput. Harmon. 46, 154-176 (2019)

Publisher's Note Springer Nature remains neutral with regard to jurisdictional claims in published maps and institutional affiliations. 